\title{
Human Face Image Segmentation using Level Set Methodology
}

\author{
M.Kumaravel \\ Embedded Systems,
}

\author{
S.Karthik \\ Centre for Excellence \\ in Computational \\ Engineering and \\ Networking, \\ Amrita Vishwa Vidhyapeetham, Coimbatore 641112 \\ P.Sivraj \\ Dept of EEE,
}

K.P.Soman

Centre for Excellence

in Computational

Engineering and

Networking,

\begin{abstract}
Face segmentation plays an important role in various applications such as human computer interaction, video surveillance, biometric systems, and face recognition for purposes including authentication and authorization. The accuracy of face classification system depends on the correctness of segmentation. Robustness of the face classification system is determined by the segmentation algorithm used, and the effectiveness in segmenting images of similar kind. This paper explains the level set based segmentation for human face images. The process is done in two stages: In order to get better accuracy, binarization of the image to be segmented is performed. Next, segmentation is applied on the image. Binarization is the process of setting pixel intensity values greater than some threshold value to "on" and the rest to "off". This process converts the input image into binary image which is used for segmentation. Second process is image segmentation for eliminating the background portion from the binarized image which is obtained after the binarization of the original image. Conventional approaches use separate methods for binarization and segmentation. In this paper we investigate the use of recently introduced convex optimization methods, selective local/global segmentation (SLGS) algorithm [16] for simultaneous binarization and segmentation. The approach is tested in MATLAB and satisfactory results were obtained.
\end{abstract}

\section{Keywords}

Level Set, Face Recognition, Face Classification, Active Contours, Binarization, Segmentation

\section{INTRODUCTION}

Image segmentation splits a given image into number of regions which is of interest. This helps to solve a number of computer vision problems. Number of methods are devised and proposed by researchers in recent years each with varying and varied level of applications. Image segmentation is one of the challenging tasks based on several reasons. The generalization of algorithm does exists with a number of user specified parameters, which has efficiency and effectiveness limited based on the number of parameters specified. Noise, inhomogeneity, acquisition artifacts and poor contrast add up to the problem for unguided segmentation.

Human face segmentation is one of the active research area in image processing which finds its applications in a number of fields including face identification systems, face tracking, video surveillance and security control system, authentication systems and human computer interface. The applications of face recognition to produce the desired results have to be given with perfectly segmented image. The notable factors influencing the segmentation are complex background color, skewness, orientation, and the distance of the device from the human face.

Recently developed methods for human face segmentation are neural networks [10], Eigen faces with background learning [13], statistical approach [14], fuzzy pattern matching [11] and color information and geometric knowledge as discussed by Rudy Adipranata et al [12]. The main goal of face segmentation is to identify if, there exists a face in a given image and to extract the same for processing. The various factors associated with face segmentation are Intensity, Pose, Structural components, Image rotation, Facial expression, Poor quality, Occlusion and Illumination.

The different types of face segmentation methods are based on geometry, color, appearance, motion and edge information. Mostly segmentation is performed based on changes on values of intensity, which leads to misclassification in cases where the intensities of the background are more than the object to be segmented on color based segmentation methods.

Binarization involves assigning a value of ' 0 ' or ' 1 ' with threshold values as mean value of the pixel after the process of converting the same to one bit value, each from the main image. Those pixel values exceeding the threshold are given'1' and the rest are assigned ' 0 '. One of the main process of binarization, helps separating face portion pixels from the background. The process of image analysis includes conversion from gray-scale to binary image by preprocessing. Large variability of appearance of faces (pose, size), complex background, occlusions, object shadows, highlights from shiny object parts, and differences of color brightness of objects which make the problem of face image binarization on still images as hard. The techniques proposed by J.Ohya et al.[2], Y.Zhong et al.[3].,O.D.Trier et al.[4].,A.T.Abak. et al[5].,M.Sezgin et al.[6]., are usually used for image binarization.

Image Binarization methodologies that exist today are classified into two categories: Thresholding based and grouping based. When the face to be detected is well contrasted with the background most of the existing algorithms perform better, however these algorithms fail when there is no sufficient distinction between background and face to be detected [9]. Niblack's [7] and Sauvola [8] are the most widely used adaptive thresholding algorithms. The methods mentioned are robust for unevenly illuminated and varying colors than those on global ones, but they do suffer regarding the dependency of parametric values. Thresholding based methods use global or local threshold to separate text from background [9]

The global thresholding techniques convert a full image from gray values to two valued (black and white) using one threshold. If the pixel value is greater than the global 
threshold it is converted to white (background) else it is converted to black (foreground). The determination of this global threshold could be made using histogram properties, busyness properties and global edge information or determined a priori by the user.

Considering the image to gray level histogram I(x,y), which has objects darker than its background, where the object and the background pixels have gray levels which can be classified into two dominant modes. Choosing a threshold ' $\mathrm{T}$ ' we can separate the object from its background. The next is choosing an object point $(\mathrm{x}, \mathrm{y})$ which has $\mathrm{I}(\mathrm{x}, \mathrm{y})$ is greater than $\mathrm{T}$, otherwise the point chosen is called background point. Finding an intensity value separating the two peaks from the histogram is to determine the threshold $\mathrm{T}$ in the bimodal case; in normal case the high peak denotes the background (white) and the intermediate peak represents the print (black). The process of estimating the valley between the right peaks of a histogram can be tedious, in case where the histogram does not have a single pure valley between the two peaks.

Simple situations can be dealt with the global thresholding technique, one of the common issues of global thresholding occurs when the gray level transition between the object and the background is gradual and inconsistent across objects in the image. This leads to the use of local thresholding.

Mostly the pixel to be converted is examined in all global binarization methods while determining the subsequent binary value of that pixel. But in addition to this the neighborhood pixels around this pixel is also examined in local thresholding. In case the pixel selected is considerably darker than the neighborhood pixels, it is converted to black else to white. The binary value can be determined with the help of local conditions using the neighborhood information and just not based on the single threshold computed from an image-wide standpoint. The main drawback of the local methods is their high computational cost and their tendency to fail to segment the inner parts of thick characters.

Though there exist a lot of approaches for image binarization of face images. Level set framework is used to binarize the image in an optimum way, which offers an elegant way to integrate various priors. To our knowledge this work is among the one to take advantage of the level set method to achieve good results in human face image binarization.

The partitioning of a single digital image into multiple segments refers to segmentation. The aim of segmentation is to make the image that is easily understood by the machine for processing. The segmentation helps identify object and object boundary like lines, curves, etc., that are present on the image.

In this paper, we explain the use of Level Set based face segmentation which is free from the drawbacks of the previous methods. The process uses signed pressure force for evolving contours and edge stopping functions for stopping the curve at the boundaries.

\section{THEORY BEHIND THE METHOD}

The method uses optimization theory and level set theory to do segmentation. Image binarization and segmentation can be done using Variational methods. Selective local or global segmentation algorithm [16] is used in this paper which is considered as simple and fast while existing techniques require separate methods for binarization and segmentation.

\subsection{Level Set Theory}

The level set method is a numerical technique used for tracking interfaces and shapes. Level set is optimization method to extract or segment the object by its shape from an image. These interface can have sharp corners . The technique can find a wide range of application including problems in image processing, computer graphics, shape of snowflakes.

Consider an image $\mathrm{f}$ with background and foreground. Boundaries can be detected using curve evolution. The boundary of an open domain can be represented using a curve $\mathrm{C}$ as the isoline of a Lipschitz continuous function:

$$
f: \Omega \rightarrow R
$$

$\phi: \Omega \rightarrow C$, where $C=\{(x, y) \in \Omega: \phi(x, y)=0\}$

For a general curve $\mathrm{C}, \phi$ it can be defined as a signed distance function to $\mathrm{C}$

$$
\phi(x, y)= \begin{cases}\operatorname{dist}((x, y), C) & \text { if }(x, y) \in \text { inside } C \\ -\operatorname{dist}((x, y), C) & \text { if } \quad(x, y) \in \text { outside } C \\ 0 & \text { if } \quad(x, y) \in C\end{cases}
$$

We have to operate on the family of curves $C(t)$ over the time $\mathrm{t} \geq 0$ to perform curve evolution.

\subsection{Active Contours}

Active contour is the process of curve fitting iteratively to an image based on its shapes and the image values until it stabilizes. Two types of active contour curves are used to fit images. They are parametric and geometric active contours. Parametric active contours are used to store vertices and each of vertexes can be moved iteratively. Geometric active contours are stored as coefficients and new coefficients are computed. Geometric active contours have many advantages over parametric active contours, such as computational simplicity and the ability to change curve topology during deformation

\subsubsection{Active Contour models}

They are based on two criterions namely regularization of the curve and stopping criterion. It can be used for boundary detection. There are two types of models edge based models and region based.

\subsubsection{Edge based models}

This model divides the image based on the discontinuities in image $\mathrm{f}$. An edge function $\mathrm{g}(\mathrm{x}, \mathrm{y})$ is defined which has a value close to zero near edges and large value away from the edges. It is given by (1). 


\begin{tabular}{|rrrrrrrr|}
\hline 1 & 1 & 1 & 1 & 1 & 1 & 1 & 1 \\
1 & 1 & 1 & 1 & 1 & 1 & 1 & 1 \\
1 & 1 & 1 & 0 & 0 & 1 & 1 & 1 \\
1 & 1 & 0 & 0 & 0 & 0 & 1 & 1 \\
1 & 1 & 0 & -1 & -1 & 0 & 0 & 1 \\
1 & 0 & 0 & -1 & -1 & -1 & 0 & 1 \\
1 & 0 & -1 & -1 & -1 & -1 & 0 & 1 \\
1 & 0 & -1 & -1 & -1 & -1 & 0 & 1 \\
1 & 0 & -1 & -1 & -1 & 0 & 0 & 1 \\
1 & 0 & -1 & -1 & -1 & 0 & 1 & 1 \\
1 & 0 & 0 & -1 & 0 & 0 & 1 & 1 \\
1 & 1 & 0 & 0 & 0 & 1 & 1 & 1 \\
1 & 1 & 1 & 1 & 1 & 1 & 1 & 1 \\
1 & 1 & 1 & 1 & 1 & 1 & 1 & 1 \\
1 & 1 & 1 & 1 & 1 & 1 & 1 & 1
\end{tabular}

Fig 1: The Level Set Visualization

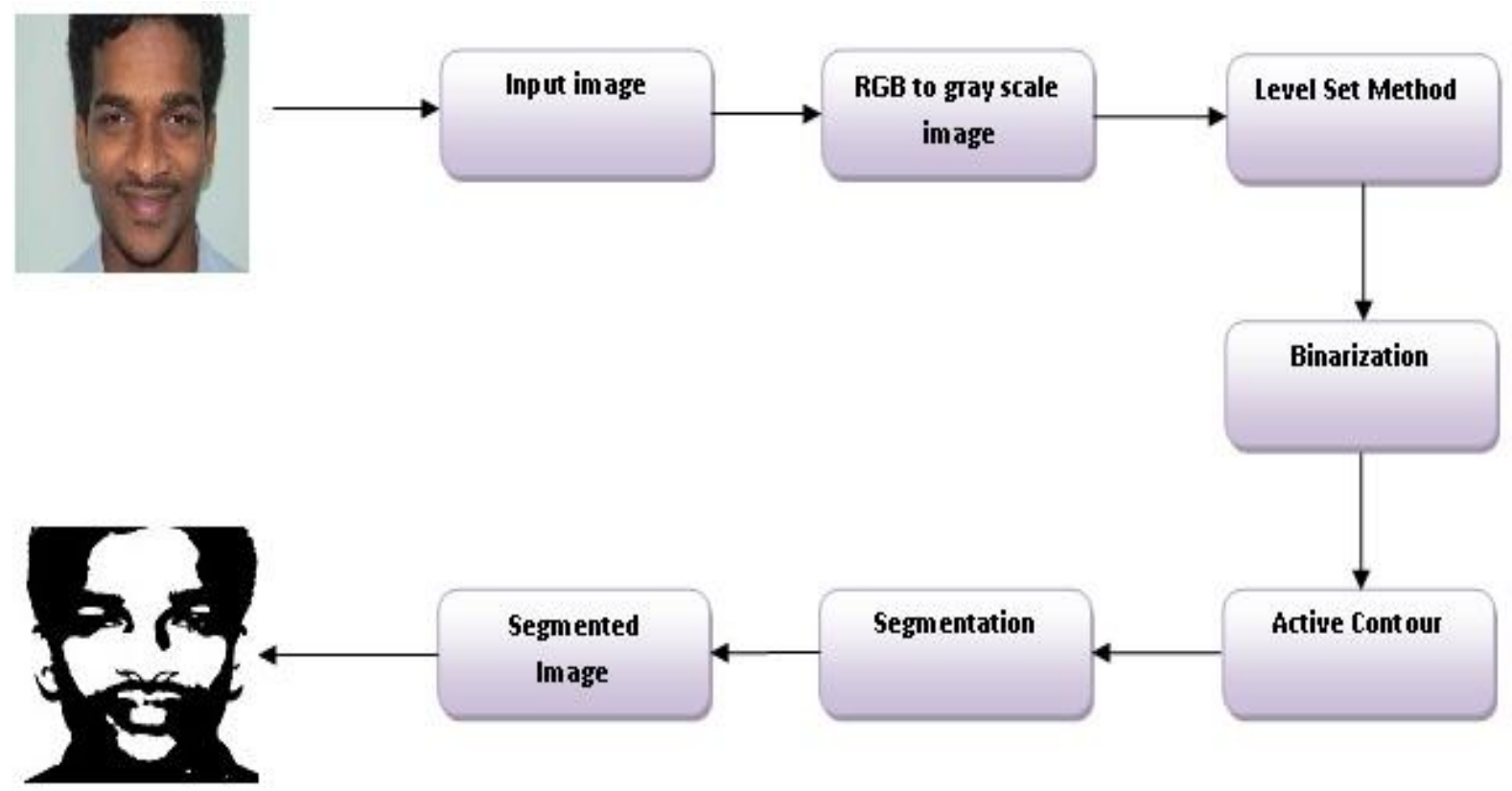

Fig 2: The System Block Diagram of Binarization and Segmentation

$$
\begin{aligned}
& g(x, y)=\frac{1}{1+\mid \nabla\left(\left.G_{\sigma} * f(x, y)\right|^{2}\right.} \\
& \min _{\phi}\left\{J(\phi)=\int_{\Omega} g(x, y) \mid \nabla H(\phi(x, y) \mid d x d y .\right.
\end{aligned}
$$

\subsubsection{Region Based Models.}

Region based methods segment an image into objects and background. This is done on the basis of similarity of intensity of pixels [16],[17] . Here the following energy is minimized:
Geodesic active contours, an edge based model proposed by Caseless et al [20], Kichenassamy et al [19] is

$$
\begin{aligned}
\min _{\mathrm{C}, \mathrm{c}_{1}, \mathrm{c}_{2}} \mathrm{E}\left(\mathrm{C}, \mathrm{c}_{1}, \mathrm{c}_{2}\right)= & \int_{\text {inside }(\mathrm{C})}^{\int}\left(\mathrm{f}-\mathrm{c}_{1}\right)^{2} \mathrm{dxdy} \\
& +\int_{\text {outside }(\mathrm{C})}\left(\mathrm{f}-\mathrm{c}_{2}\right)^{2} \mathrm{dxdy} \\
& +\lambda \operatorname{Length}(\mathrm{C})
\end{aligned}
$$


$H(\phi)=\left\{\begin{array}{l}1 \text { if } \phi \geq 0 \\ 0 \text { if } \phi<0\end{array}\right.$,

and the delta function

$\delta(\phi)=H^{\prime}(\phi)$

in the weak sense.

In the implicit representation the minimization problem can be represented as:

$$
\begin{aligned}
\min _{\mathrm{f}, \mathrm{c}_{1}, \mathrm{c}_{2}} \mathrm{E}\left(\mathrm{C}, \mathrm{c}_{1}, \mathrm{c}_{2}\right)= & \int_{\Omega}\left(\mathrm{f}-\mathrm{c}_{1}\right)^{2} \mathrm{H}(\mathrm{f}) \mathrm{dxdy} \\
& +\int_{\Omega}\left(\mathrm{f}-\mathrm{c}_{2}\right)^{2}(1-\mathrm{H}(\mathrm{f})) \mathrm{dxdy} \\
& +\lambda \int_{\Omega}|\tilde{N} \mathrm{H}(\mathrm{f})|
\end{aligned}
$$

and the inside means $\mathrm{c} 1$ and outside mean $\mathrm{c} 2$ is obtained as:

$$
\begin{aligned}
& c_{1}=\frac{\int_{\Omega} f(x, y) H(\phi) d x d y}{\int_{\Omega} H(\phi) d x d y}, \\
& c_{2}=\frac{\int_{\Omega} f(x, y)(1-H(\phi)) d x d y}{\int_{\Omega}(1-H(\phi)) d x d y}
\end{aligned}
$$

For minimization in

$$
\phi, \frac{\partial \phi}{\partial \mathrm{t}}=-\frac{\partial E}{\partial \phi}
$$

is used. Simplification gives:

$$
\phi(x, y, 0)=\phi_{0}(x, y),
$$

$$
\frac{\partial \phi}{\partial t}=\delta(\phi)\left[-\left(f-c_{1}\right)^{2}+\left(f-c_{2}\right)^{2}+\lambda K(x, y)\right]
$$

Level Set Method can solve (6) but it gives only a local minimizer because its energy is non-convex. This model is less dependent on initial curve but if the initial contour is far away from object it may not extract it. The active contour method tries to convexify the energy and minimize it to compute a global minimizer.

\subsubsection{Signed Pressure Force (SPF)}

The SPF function [16] is given as:

$$
\operatorname{spf}(f(x, y))=\frac{f(x, y)-\frac{c_{1}+c_{2}}{2}}{\max \left(\left|f(x, y)-\frac{c_{1}+c_{2}}{2}\right|\right)}, x \in \Omega
$$

$$
\operatorname{Min}(f(x, y))<\frac{c_{1}+c_{2}}{2}<\operatorname{Max}(f(x, y)), x \in \Omega
$$

The SPF function is substitute for EFS function in Level Set function, which controls the

\section{RECENT APPROACHES}

\subsection{Block Diagram}

For image segmentation and binarization, we make use of Active Contour Method (ACM) and Level set method. Active contour models are used to identify the object boundaries of an image. The contours evolve and fit on to the boundaries of the face, this helps us easily binarize the image. We have marked the contours with green and given the labels $\mathrm{C} 1, \mathrm{C} 2$, C3, C4, for each contour for better visualization. The outer contour $\mathrm{C} 1$ gives the boundary of the face. It is the longest contour. The contours $\mathrm{C} 2, \mathrm{C} 3$, and $\mathrm{C} 4$ correspond to the contours drawn for each loop in the face portion. Contours at the boundaries of the face are equivalent to getting the face segmented. So by applying the active contour models we can do binarization and segmentation simultaneously

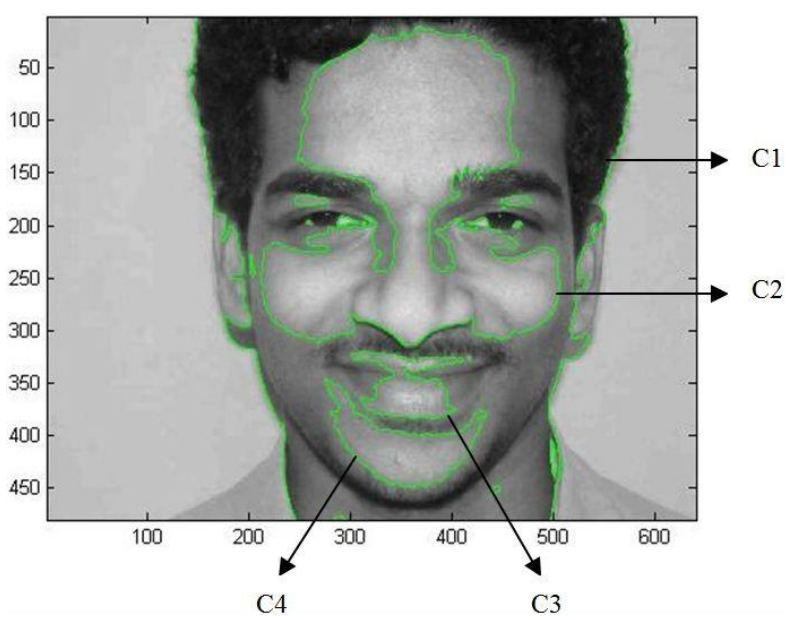

Fig 3: Contour Fitting operation diagram

\section{EXPERIMENTS AND RESULT}

The present paper apart from experimentation of the methods performs binarization and face segmentation. The algorithm was tested on face images, SLGS method is used for facial image analysis. Analysis shows that selective local or global segmentation method (SLGS) is better than the Fast global minimization algorithm (FGM). SLGS algorithm though it takes time, but performs better segmentation.

\subsection{Selective local or global segmentation algorithm}

The robustness of segmentation is completely dependent on the contours that fit the object boundary under consideration. Models utilizing the region information inside and outside of the contours are less influenced by noise and tend to provide better results for images even with weak boundaries. Notable methods of this type are $\mathrm{C}-\mathrm{V}$ model [19], which has its derivation from Mumford-Shah segmentation techniques [20]. 


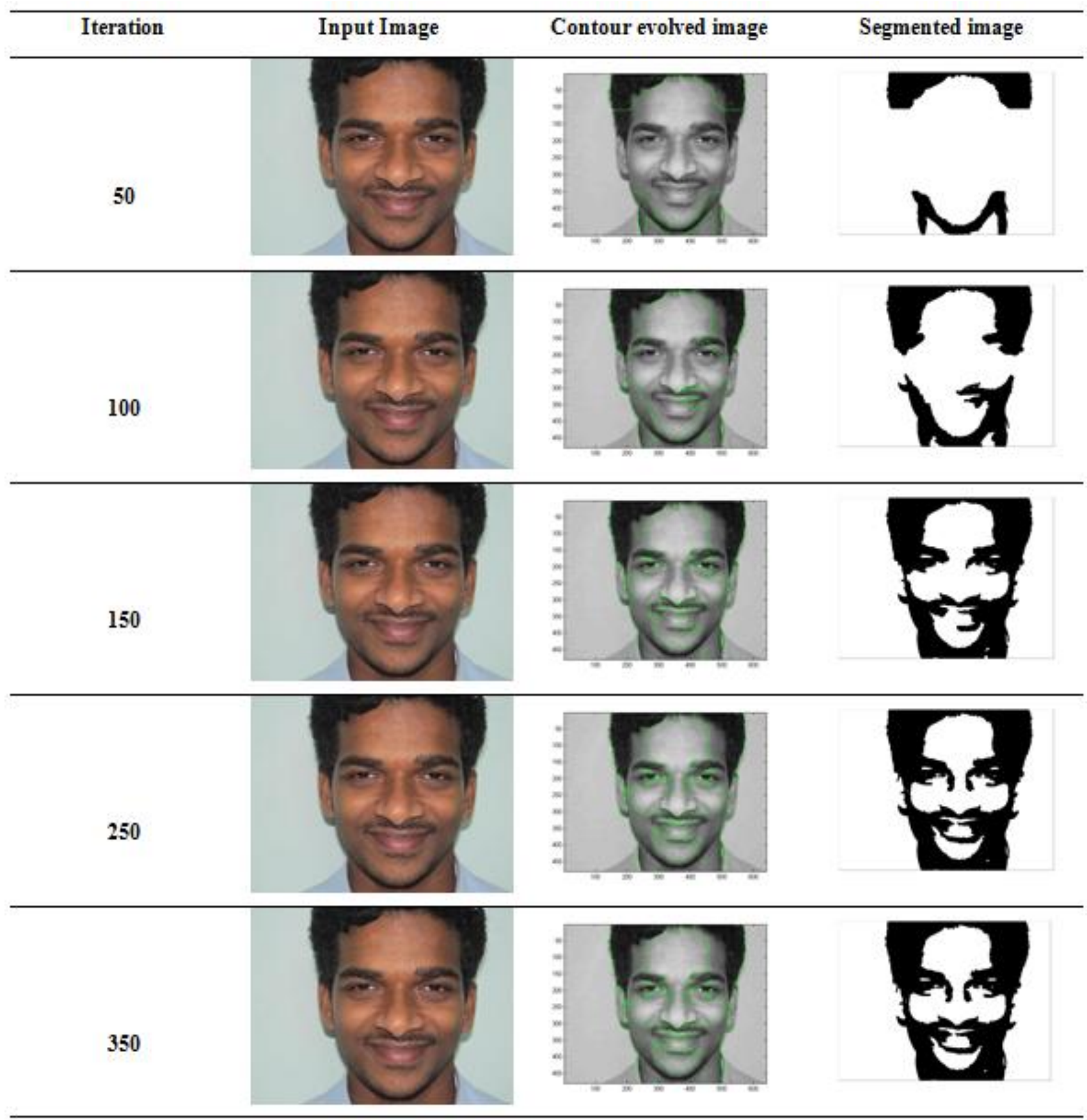

Fig 4: Results for different iteration levels and corresponding segmented images for test image 1. 


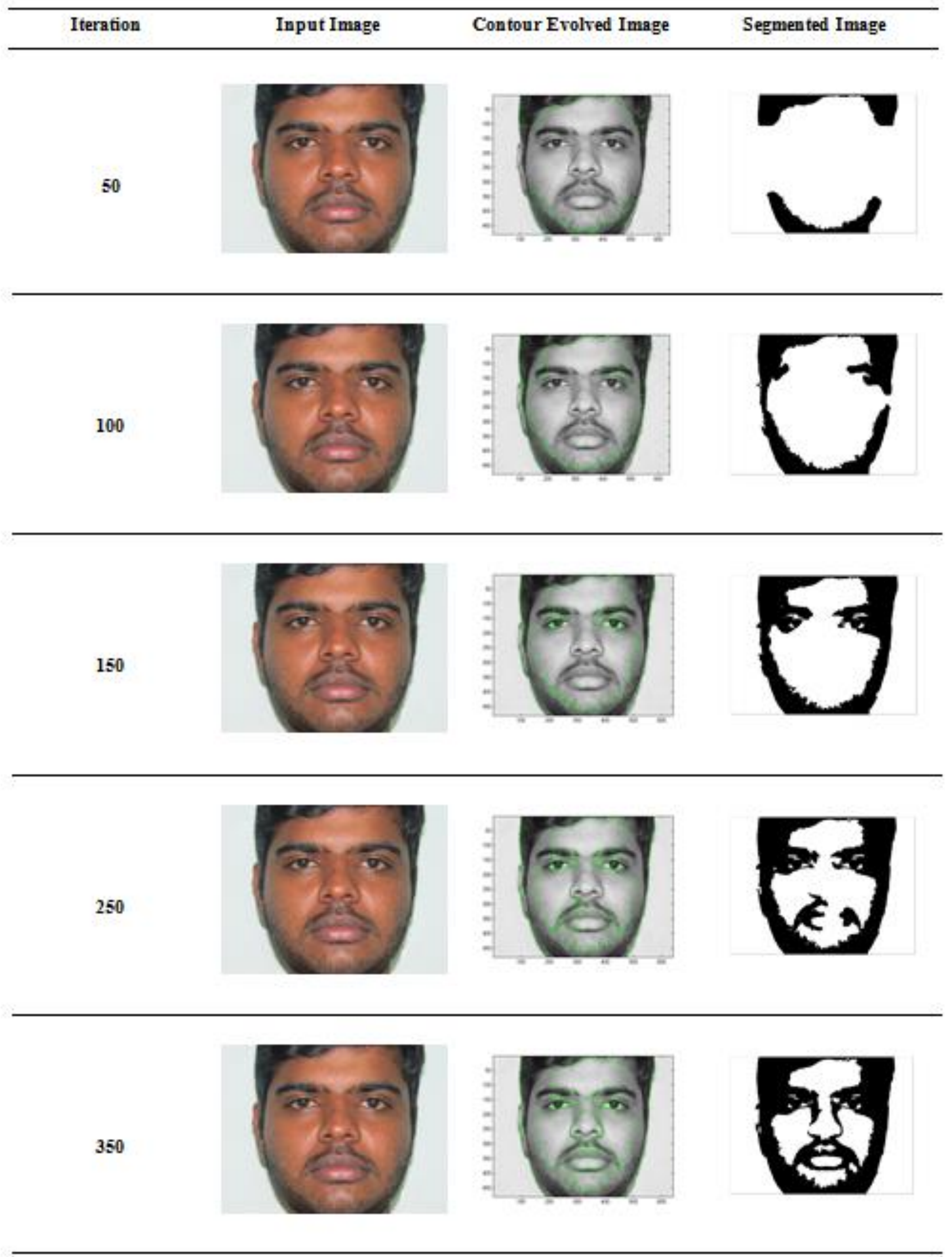

Fig 5: Results for different iteration levels and corresponding segmented images for test image 2 
Start and place of initial contours never account for segmentation using C-V model [19] which is one of the best notable global segmentation model. Instead, the GAC Model [18] is dependent on the initial contour information but its segmentation is more near similar representation of the object under consideration. GAC model suits best for selective local segmentation.

Zhang, K et al., [16] proposed a new region based ACM that combines the best properties of $\mathrm{C}-\mathrm{V}$ and GAC models. This method uses the statistical information inside and outside the contour in order to construct the signed pressure force (SPF) function [21] used to control the contour evolution.

This property of contour controlling using the SPF makes it suitable for face segmentation and the results are as expected and shown in Figure 4 and Figure 5. In this method the initialization of contour takes only once where the algorithm takes care of handling all the objects in the images, whereas other similar methods require contour initialization for each interior objects.

Human face segmentation is one which involves segmenting and extracting the face portion from images which has possibilities to have a number of objects. These objects, present in the image makes the segmentation algorithm to identify them too. But the actual intelligence of this algorithm uses both selective local or global segmentation algorithm which makes it robust for face segmentation.

The initial contour place and start independence, of $\mathrm{C}-\mathrm{V}$ model helps to identify the face portion in the image with other objects. The fine fitting of the contour by the use of SPF helps to exactly fit the contour on the face portion in the image. The SPF function has opposite signs around the boundary. If the contour is inside the face portion the SPF has negative sign and this in turn expand the contour to move towards the face boundary. If the contour is outside the face portion the SPF has positive sign and will move the contour towards the face boundary. Thus the contour fits on to the face and gives good segmentation results.

\section{CONCLUSION}

This method stays as the pre-processing stage for image processing tasks and security authentication using human face image. This experimented method using active contour based selective local or global segmentation also performs Binarization. This method employs signed pressure force as edge stopping function to fit the contour on the human face image. This method has to be fine-tuned for near similar images which does account for future work on the same.

\section{REFERENCES}

[1] S Karthik et al., Level Set Methodology for Tamil Document Image Binarization and Segmentation. International Journal of Computer Applications 39(9):712, February 2012. Foundation of Computer Science, New York, USA

[2] J. Ohya, A. Shio, and S. Akamatsu. "Recognizing characters in scene images". IEEETrans. Pattern, Anal.Mach.Intell., 16(2), 1994, pp.214-220.

[3] Y. Zhong, K. Karu, and A.K. Jain. "Locating text in complex color images.", Proc. of 3rd Int. Conf. Document Analysis and Recognition, 1995, 146 - 149 vol.1.

[4] O. D. Trier and T.Taxt, "Evaluation of binarization methods for document images",. IEEE Trans. Pattern Anal. Machine Intell., vol. 17, Mar. 1995, pp. 312-315.

[5] A.T. Abak, U. Baris, and B. Sankur, "The Performance Evaluation of Thresholding Algorithms for Optical
Character Recognition”, ICDAR 97, Ulm, Germany, 1997, pp. 697-700.

[6] M. Sezgin, "Survey over image thresholding techniques and quantitative performance evaluation", Journal of electronic imaging, 13, 146, 2004 doi:10.1117/1.1631315.

[7] W. Niblack, "An Introduction to Image Processing", Prentice-Hall, En- glewood Cliffs, NJ 1986, pp. 115116.

[8] J. Sauvola and M. Pietaksinen, "Adaptive document image binarization," Pattern Recogn. 33, 2000, pp 225236.

[9] Basura Fernando, Sezer Karaoglu, Alain Trmeau, "Extreme Value Theory Based Text Binarization In Documents and Natural Scenes", 3rd Int. Conf. Machine Vision, Hong Kong, 2010

[10] H.A. Rowley, S. Baluja and T.Kanade, "Rotation Invariant Neural Network-Based Face Detection“, Proc IEEE Conf. Computer Vision and Pattern Recognition, 1998, pp 38-44.

[11] H. Wu, Q. Chen, M. Yachida, "Face Detection from Color Images using a Fuzzy pattern Matching Method", IEEE Trans. Pattern Analysis and Machine Intelligenc, vol. 21, no. 6, 1999, 557-563.

[12] Rudy Adipranata et al.," Fast Method for Multiple Human Face Segmentation in Color Image", International Journal of Advanced Science and Technology, Vol. 3, February, 2009

[13] R. K. Singh and A. N. Rajagopalan, "Background learning for robust face recognition", Int'l Conference on Pattern Recognition, 2002.

[14] H.A. Rowley, S. Baluja and T.Kanade, "Rotation Invariant Neural Network-Based Face Detection", Proc IEEE Conf. Computer Vision and Pattern Recognition, 1998, pp 38-44.

[15] X. Bresson, S. Esedo, P. Vandergheynst, J.-philippe Thiran, and S. Osher, "Fast Global Minimization of the Active Contour / Snake Model," Journal of Mathematical Imaging and Vision, 2007 28: 151167.

[16] Zhang, K., Zhang, L., Song, H., and Zhou, W. (2010). "Active contours with selective local or global segmentation: A new formulation and level set method.", Image and Vision Computing 28(4), 668-676. Elsevier B.V. doi: 10.1016/j.imavis.2009.10.009

[17] David Rivest-Hnault , Reza Farrahi Moghaddam, Mohamed Cheriet "A local linear level set method for the binarization of degraded historical document images", Springer-Verlag 2011

[18] V. Caselles, R. Kimmel, G. Sapiro, Geodesic active contours, in: Processing of IEEE International Conference on Computer Vision'95, Boston, MA, 1995, pp.694-699.

[19] T. Chan, L. Vese, Active contours without edges, IEEE Transaction on Image Processing 10 (2) (2001) 266-277.

[20] D. Mumford, J. Shah, Optimal approximation by piecewise smooth function and associated variational problems, Communication on Pure and Applied Mathematics 42 (1989) 577-685.

[21] C.Y. Xu, A. Yezzi Jr., J.L. Prince, On the relationship between parametric and geometric active contours, in: Processing of 34th Asilomar Conference on Signals Systems and Computers, 2000, pp. 483-489.

[22] S. Osher, R. Fedkiw, Level Set Methods and Dynamic Implicit Surfaces, Springer Verlag, New York, 2002. 